\title{
American Psychological Foundation Donors
}

APF extends its thanks to all of its donors over the past year. Those who made gifts of $\$ 1,000$ or more in the past year are acknowledged by name below.

Founders (\$250,000 and Above)

Franklyn Springfield Charitable

Remainder Unitrust

\section{Stakeholders (\$100,000-\$249,999)}

Dr. Diana Slaughter Kotzin and Mr. Joseph Kotzin

Pioneers (\$50,000-\$99,999)

Dr. Marian R. Stuart

\section{Trendsetters (\$25,000-49,000)}

GAPPP

Dr. Bradley and Dr. Keith Marshland

Initiators (10,000-24,999)

The Estate of. Mary Gratz

The Estate of Frances D. Horwitz, PhD

\section{Advocates (\$5000-\$9999)}

Academy for Cancer Wellness

Margaret and Paul Baltes Foundation

Dr. Louise Douce

Mrs. Sandra M. Fowler

Dr. Patricia A. Stark

Dr. Mary G. Yancy

\section{Proponents (\$1,000-\$4,999)}

APA Division 29- Society for the Adcance of Psychotherapy

Steven D. Axelrod, PhD

J. Gayle Beck, PhD

Sharon Berry, PhD

Dr. Ronald T. Brown

Dr. Kathleen S. Brown

Dorothy W. Cantor, PsyD

Dr. John C. Cavanaugh

Dr. Diana M. Concannon

Dr. Joan M. Cook

Dr. Helen L. Coons

Andrea Corn, PsyD

Dr. Jaine Darwin

Dr. Rosie Phillips Davis and Mr. John

Davis
Dennis Debiak, PsyD

Leadership Institute for Women

Dr. Chanming Duan and Dr. Lizhao Wang

Dr. Anne M. Etgen

Dr. Linda M. Forrest and Dr. Warren

Holmes

Dr. Michael A. Goldberg

Dr. Richard R. Hansen

Dr. John F. House

Dr. Elliot Jurist

Debra M. Kawahara, PhD

Terrence M. Keane, PhD

Dr. Russell B. Lemle

Dr. Paul and Mrs. Wendy Leung

Dr. Jana N. Martin

Susan H. McDaniel, PhD, ABPP

Dr. Susan McPherson

Dr. Nancy R. McWillliams

Dr. Jeffrey S. Mio

Dr. Olivia Moorehead-Slaughter

Bernard F. Natelson, PsyD and Lisa

Raufman, EdD

Dr. Genevieve S. Neal-Perry

Dr. Paul D. Nelson

Dr. Katerine C. Nordal

Oxford University Press

Dr. Natalie Porter

Dr. Linda Richardson and Dr. Rodney

Lowman

Beth N. Rom-Rymer, PhD

Mr. Alan Routh

Joan Sarnat, PhD

Dr. Joseph G. Schaller

Dr. Sandra L. Shullman and Dr. Peg

Mosher

Dr. Barbara A. Van Horne

Dr. Jonathan and Mrs. Carolyn Vitriol

Mr. Charles Weaver, Sr and Mrs. Jeanette

Weaver

Wayne Weiten, $\mathrm{PhD}$

Workday

For more information about APF, please visit www.apa.org/apf. 